(1)

CrossMark

\title{
Demystifying fibrotic hypersensitivity pneumonitis diagnosis: it's all about shades of grey
}

\author{
Simon L.F. Walsh ${ }^{1}$ and Luca Richeldi ${ }^{2}$ \\ Affiliations: ${ }^{1}$ National Heart and Lung Institute, Imperial College, London, UK. ${ }^{2}$ Fondazione Policlinico \\ A. Gemelli IRCCS, Università Cattolica del Sacro Cuore, Rome, Italy. \\ Correspondence: Simon L.F. Walsh, National Heart and Lung Institute, Imperial College, London SW3 6LY, \\ UK. E-mail: slfwalshdgmail.com
}

@ERSpublications

The headcheese pattern which describes a form of mosaic attenuation which combines areas of ground-glass opacification, lobular areas of low attenuation and normal lung is highly specific for a diagnosis of fibrotic hypersensitivity pneumonitis http://bit.ly/2WO2mn7

Cite this article as: Walsh SLF, Richeldi L. Demystifying fibrotic hypersensitivity pneumonitis diagnosis: it's all about shades of grey. Eur Respir J 2019; 54: 1900906 [https://doi.org/10.1183/13993003.00906-2019].

The spotlight on diagnosis in hypersensitivity pneumonitis (HP) intensifies. Over the past 18 months we have seen two pulmonary perspectives proposing diagnostic approaches to HP; an international Delphi survey and several imaging studies focused on clinical and imaging features predictive of HP; and clinical practice guideline initiatives for HP, sponsored by the American Thoracic Society and American College of Chest Physicians, are underway [1-5]. Despite this growing literature, HP continues to resist our best attempts to develop an accurate, reproducible case definition for this disorder. Making a diagnosis of HP is challenging because it is often shadowed by marginal exposure histories, borderline antigen positivity or discordant imaging patterns, and treatment decisions are often based on diagnostic probability rather than certainty $[4,5]$. As in idiopathic pulmonary fibrosis (IPF), chest high-resolution computed tomography (HRCT) plays a central role in the initial evaluation of HP and involves the identification of specific patterns. For many years, the presence of mosaic attenuation (MA) on CT has been widely accepted as a cardinal imaging feature of HP.

In 2008, the Fleischner Society Glossary of Terms for Thoracic Imaging defined MA as a patchwork of regions of differing density caused by 1) air trapping secondary to bronchial or bronchiolar obstruction or 2) interstitial lung disease characterised by ground-glass opacity; in this situation, areas of higher attenuation represent the interstitial process and areas of lower attenuation represent the normal lung [6]. In the same year, SILVA et al. [7] reported that MA (described at lobular areas with decreased attenuation and vascularity in their study) was a distinguishing feature of fibrotic hypersensitivity pneumonitis (FHP) and particularly useful for separating this disorder from its key imaging differentials, usual interstitial pneumonia (UIP) and fibrotic non-specific interstitial pneumonia. Therefore, in fibrotic lung disease, the presence of MA on CT has particular diagnostic importance. Our faith in this sign as an arbiter between IPF and FHP is reflected in its longevity as a non-IPF CT pattern in almost a decade of international IPF guideline statements. In 2011, the joint IPF guideline listed the presence of "diffuse" MA/air trapping as inconsistent with UIP [8]. The latest iteration of this guideline indicates that "marked" MA on CT should alert the clinician that an alternative diagnosis to IPF is likely [9]. Finally, in a White Paper published by the Fleischner Society, "extensive" MA is described as a CT feature most consistent with a non-IPF diagnosis, in this case, FHP [10]. In this issue of the European Respiratory Journal, BARNETT et al. [11] challenge our faith. 
The premise of this study is that three different radiological patterns result in an MA appearance on CT; namely, lobules of low attenuation lung reflecting air trapping with normal adjacent lung, patchy ground-glass opacities with spared, healthy lung and a third variation where ground-glass opacities and lobules of low attenuation coexist, called the headcheese sign (figure 1) [12]. The authors argue that by amalgaming these patterns together, the diagnostic value of each feature individually is obscured. Although it is widely accepted that the presence of MA in fibrotic lung disease has diagnostic value, the performance characteristics of each pattern captured by this term are unknown BARNETT et al. [11] address this shortfall in a cohort of patients with multidisciplinary diagnoses of IPF and FHP using different thresholds specified by the Fleischner Society IPF guidelines and the 2018 joint IPF guideline statement. The key finding was that the headcheese sign, when present in three or more lobes, provided the optimum balance between sensitivity (0.49) and specificity (0.93) for FHP. In contrast, although expiratory air trapping and low-density lobules on inspiratory images were sensitive for FHP ( 0.82 and 0.78 , respectively), neither matched the headcheese sign for specificity ( 0.54 and 0.49 , respectively).

A strength of this study is its derivation-validation cohort design which helps to prevent spurious conclusions being drawn from models that are overfitted on a single population. Equally important is its clinical utility since diagnostic algorithms based on HRCT patterns are notoriously vulnerable to low levels of interobserver variability $[13,14]$. The high levels of interobserver agreement reported in the study are encouraging; $\mathrm{k}=0.65$ for both the presence of low-density lobules and air trapping and $\mathrm{k}=0.48$ for preserved lobules. However, these results reflect the scoring of CT patterns by two expert radiologists; how consistently less experienced radiologists apply these signs is yet to be seen.

Several significant ancillary findings emerge from this study. Ironically, the study that contributed most to enshrining the status of MA as inconsistent with UIP also reported MA in 43\% of IPF participants, a finding that is widely overlooked [7]. In the study by BARNETT et al. [11], this observation is corroborated;

a)

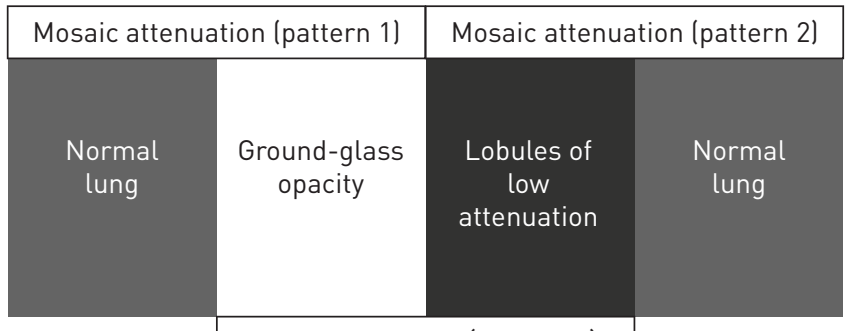

Headcheese sign (pattern 3)

b)

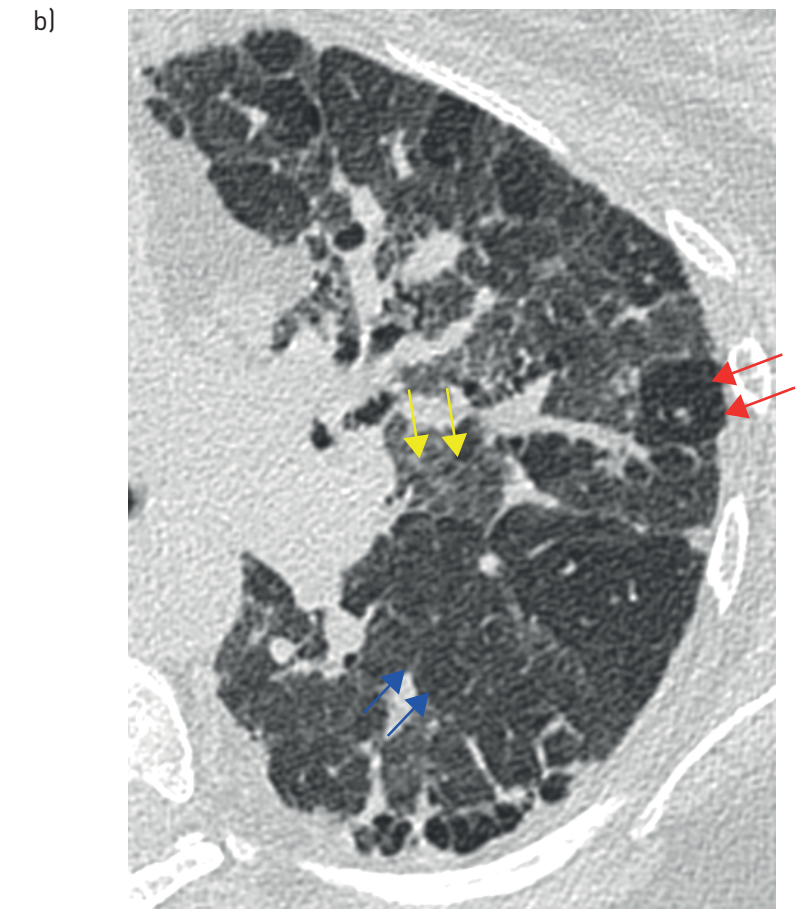

FIGURE 1 a) Schematic illustrating the three different radiological patterns that produce mosaic attenuation on computed tomography; lobules of low attenuation lung reflecting air trapping with normal adjacent lung, patchy ground-glass opacities with spared, healthy lung and ground-glass opacities coexisting with lobules of low attenuation called the headcheese sign. b) Cropped axial high-resolution computed tomography image in a patient with fibrotic hypersensitivity pneumonitis. Red arrows indicate lobules of low attenuation lung reflecting air trapping; blue arrows indicate normal lung; and yellow arrows indicate areas of ground-glass opacity. 
more than $50 \%$ of IPF patients in the derivation cohort had at least one low-density lobule in three or more lobes. Also, air trapping was present in $46 \%$ of IPF cases on expiratory CT. These findings should not be surprising; airway exposures are common in IPF patients due to smoking or, possibly, gastroesophageal reflux $[15,16]$. Importantly, no extent of MA completely excluded IPF, strongly arguing that MA can no longer be considered incompatible with UIP on CT. Second, rather than attempting to distil diagnostic patterns of HP (i.e. fibrotic and non-fibrotic forms combined), BARNETT et al. [11] adopted a pragmatic approach by evaluating CT patterns that separate FHP from its usual differential diagnosis, IPF. Cases included in the study were based on multidisciplinary diagnoses; therefore, these findings apply to patients where FHP and IPF are the primary differential diagnoses - a frequent clinical dilemma [17]. Their findings reinforce the view that since fibrotic and non-fibrotic HP have separate and distinct diagnostic pathways, any useful classification of HP should begin with this dichotomous separation.

The study also highlights the importance of semantic precision. Simplicity can be a good thing; we are all familiar with the "KISS" principle ("Keep It Simple, Stupid"), which states that most things work best when they are kept simple rather than made complicated. But in language, simplicity can also flatten meaning. In the case of MA, BARNETT et al. [11] eloquently demonstrate that reducing distinct CT patterns to a single entity deprives the radiologist (and their multidisciplinary colleagues) of meaningful diagnostic information. The presence of MA is an often hotly debated observation in patients with fibrotic lung disease, and it is interesting to note, that at the authors' institution, which is well-known worldwide for its expertise in interstitial lung disease, MA is given little weight when constructing a diagnosis. These data reinforce the importance of making distinctions between different types of MA and provide radiologists with a specific imaging target to aim for, namely, the headcheese sign when confronted with the FHP/UIP challenge. The Fleischner Glossary of Terms for Thoracic Imaging provides radiologists with a toolbox for conveying interpretation and formulating a diagnosis. The study by BARNETT et al. [11] reminds us of the need to chisel and sharpen our tools for clarity and diagnostic precision.

Conflict of interest: S.L.F. Walsh reports personal fees for lecturing from Boehringer Ingelheim, Roche, Intermmune, Sanofi-Genzyme and Bracco, personal fees for advisory board work from Boehringer Ingelheim and The Open Source Imaging Consortium, outside the submitted work. L. Richeldi reports personal fees for consultancy from Sanofi-Aventis, ImmuneWorks, Celgene, Nitto, Bristol Myers Squibb and Pliant Therapeutics, grants and personal fees for advisory board work from Roche, personal fees for lecturing from Shionogi, grants and personal fees for steering committee work from Boehringer Ingelheim, personal fees for advisory board work from Fibrogen and Promedior, personal fees for editorial activity from DynaMed, outside the submitted work.

\section{References}

1 Johannson KA, Elicker BM, Vittinghoff E, et al. A diagnostic model for chronic hypersensitivity pneumonitis. Thorax 2016; 71: 951-954.

2 Morisset J, Johannson KA, Jones KD, et al. Identification of diagnostic criteria for chronic hypersensitivity pneumonitis: an international modified Delphi survey. Am J Respir Crit Care Med 2017; 197: 1036-1044.

3 Salisbury ML, Gross BH, Chughtai AR, et al. Development and validation of a radiologic diagnosis model for hypersensitivity pneumonitis. Eur Respir J 2018; 52: 1800443.

4 Salisbury ML, Myers JL, Belloli EA, et al. Diagnosis and treatment of fibrotic hypersensitivity pneumonia. Where we stand and where we need to go. Am J Respir Crit Care Med 2017; 196: 690-699.

5 Vasakova M, Morell F, Walsh S, et al. hypersensitivity pneumonitis: perspectives in diagnosis and management. Am J Respir Crit Care Med 2017; 196: 680-689.

6 Hansell DM, Bankier AA, MacMahon H, et al. Fleischner Society: glossary of terms for thoracic imaging. Radiology 2008; 246: 697-722.

7 Silva CI, Muller NL, Lynch DA, et al. Chronic hypersensitivity pneumonitis: differentiation from idiopathic pulmonary fibrosis and nonspecific interstitial pneumonia by using thin-section CT. Radiology 2008; 246: 288-297.

8 Raghu G, Collard HR, Egan JJ, et al. An official ATS/ERS/JRS/ALAT statement: idiopathic pulmonary fibrosis: evidence-based guidelines for diagnosis and management. Am J Respir Crit Care Med 2011; 183: 788-824.

9 Raghu G, Remy-Jardin M, Myers JL, et al. Diagnosis of idiopathic pulmonary fibrosis. An official ATS/ERS/JRS/ ALAT clinical practice guideline. Am J Respir Crit Care Med 2018; 198: e44-e68.

10 Lynch DA, Sverzellati N, Travis WD, et al. Diagnostic criteria for idiopathic pulmonary fibrosis: a Fleischner Society White Paper. Lancet Respir Med 2018; 6: 138-153.

11 Barnett J, Molyneaux PL, Rawal B, et al. Variable utility of mosaic attenuation to distinguish fibrotic hypersensitivity pneumonitis from idiopathic pulmonary fibrosis. Eur Respir J 2019; 54: 1900531.

12 Webb W, WaynCe R, Müller N, et al. High-resolution CT of the Lung. Wolters Kluwer/Lippincott Williams \& Wilkins, 2009.

13 Walsh SL, Calandriello L, Sverzellati N, et al. Interobserver agreement for the ATS/ERS/JRS/ALAT criteria for a UIP pattern on CT. Thorax 2015; 71: 45-51.

14 Watadani T, Sakai F, Johkoh T, et al. Interobserver variability in the CT assessment of honeycombing in the lungs. Radiology 2013; 266: 936-944.

15 Kreuter M, Raghu G. Gastro-oesophageal reflux and idiopathic pulmonary fibrosis: the heart burn in patients with IPF can no longer be silent. Eur Respir J 2018; 51: 1800921.

16 Richeldi L, Collard HR, Jones MG. Idiopathic pulmonary fibrosis. Lancet 2017; 389: 1941-1952.

17 Walsh SLF, Wells AU, Desai SR, et al. Multicentre evaluation of multidisciplinary team meeting agreement on diagnosis in diffuse parenchymal lung disease: a case-cohort study. Lancet Respir Med 2016; 4: 557-565. 